\title{
A New, High-Efficacy, Noninvasive Transcranial Electric Stimulation Tuned to Local Neurodynamics
}

\author{
[Carlo Cottone, ${ }^{1}$ Andrea Cancelli, ${ }^{1,3}$ (atrizio Pasqualetti, ${ }^{2}$ Camillo Porcaro,,${ }^{1,4,5}$ Carlo Salustri, ${ }^{1}$ \\ and ${ }^{\circ}$ Franca Tecchio ${ }^{1,6}$ \\ ${ }^{1}$ Laboratory of Electrophysiology for Translational NeuroScience, Institute of Cognitive Sciences and Technologies, Consiglio Nazionale delle Ricerche, \\ Rome, I-00185 Italy, ${ }^{2}$ Service of Medical Statistics and Information Technology, Fatebenefratelli Foundation for Health Research and Education, AFaR \\ Division, Rome, 00186 Italy, ${ }^{3} \mathrm{GMV}$, Rome, Italy, ${ }^{4}$ Movement Control and Neuroplasticity Research Group, Department of Kinesiology, KU Leuven, Leuven \\ 3001, Belgium, ${ }^{5}$ Birmingham University Imaging Centre (BUIC), School of Psychology, University of Birmingham, Birmingham, UK, and ${ }^{6}$ Catholic \\ University of the Sacred Heart
}

In this paper, we pose the following working hypothesis: in humans, transcranial electric stimulation (tES) with a time course that mimics the endogenous activity of its target is capable of altering the target's excitability. In our case, the target was the primary motor cortex (M1). We identified the endogenous neurodynamics of hand M1's subgroups of pyramidal neuronal pools in each of our subjects by applying Functional Source Separation (FSS) to their EEG recordings. We then tested whether the corticospinal excitability of the hand representation under the above described stimulation, which we named transcranial individual neurodynamics stimulation (tIDS), was higher than in the absence of stimulation (baseline). As a check, we compared tIDS with the most efficient noninvasive facilitatory corticospinal tES known so far, which is $20 \mathrm{~Hz}$ transcranial alternating current stimulation (tACS). The control conditions were as follows: (1) sham, (2) transcranial random noise stimulation (tRNS) in the same frequency range as tIDS (1-250 Hz), and (3) a low current tIDS $\left(\right.$ tIDS $\left._{\text {low }}\right)$. Corticospinal excitability was measured with motor-evoked potentials under transcranial magnetic stimulation. The mean motor-evoked potential amplitude increase was $31 \%$ of the baseline during tIDS $(p<0.001)$, and it was $15 \%$ during tACS $(p=0.096)$. tRNS, tIDS $_{\text {low }}$, and sham induced no effects. Whereas tACS did not produce an enhancement in any subject at the individual level, tIDS was successful in producing an enhancement in 8 of the 16 subjects. The results of the present proof-of-principle study showed that proper exploitation of local neurodynamics can enhance the efficacy of personalized tES.

Key words: EEG; Functional Source Separation (FSS); personal/individual dynamics of neuronal activity; primary motor area; transcranial electric stimulation; transcranial individual neurodynamics stimulation

\section{Significance Statement}

This study demonstrated that, in humans, transcranial individual neurodynamics stimulation (tIDS), which mimics the endogenous dynamics of the target neuronal pools, effectively changes the excitability of these pools. tIDS holds promise for high-efficacy personalized neuromodulations based on individual local neurodynamics.

\section{Introduction}

Previous research by Steriade and colleagues (Crochet et al., 2006) has shown that trains of stimuli delivered to local neo-

\footnotetext{
Received Aug. 3, 2016; revised July 10, 2017; accepted July 30, 2017

Author contributions: F.T. designed research; C.C. and A.C. performed research; C.C., A.C., P.P., C.P., and F.T. analyzed data; P.P., C.C., C.S., and F.T. wrote the paper.

This work was supported by Fondazione Italiana Sclerosi Multipla-Cod.2014/R (FaReMuS CuNeH) and PNRConsiglio Nazionale delle Ricerche Aging Program 2012-2018. We thank everyone involved who committed time, cooperated, and participated in this study; and Marina Di Giorgio for technical assistance.

The authors declare no competing financial interests.

Correspondence should be addressed to Dr. Franca Tecchio, Laboratory of Electrophysiology for Translational NeuroScience, Institute of Cognitive Sciences and Technologies, National Research Council (Consiglio Nazionale delle Ricerche), via Palestro 32, Rome, I-00185 Italy. E-mail: franca.tecchio@cnr.it.

DOI:10.1523/JNEUROSCI.2521-16.2017

Copyright $\odot 2018$ the authors $\quad 0270-6474 / 18 / 380586-09 \$ 15.00 / 0$
}

cortical networks of cats produce either a depression or a potentiation of the postsynaptic potentials that may last up to several minutes if the stimulation is applied at frequencies matching the individual cat's endogenous brain rhythms. More recent studies in humans have shown that the efficacy of neuromodulation of cortical regions depends on the frequency of the applied stimulation (Kanai et al., 2008; Feurra et al., 2011; Thut et al., 2011; Brinkman et al., 2016). Wide-band time-varying-current neuromodulation sustains cognitive domains more effectively than direct currents (tRNS) (Fertonani et al., 2011). Furthermore, in vitro studies have shown that both oscillatory (Fröhlich and McCormick, 2010) and scale-free (Gal and Marom, 2013) stimulations can entrain cortical physiological activities. By "entrain" here, we mean "forcing the target to change excitation." 

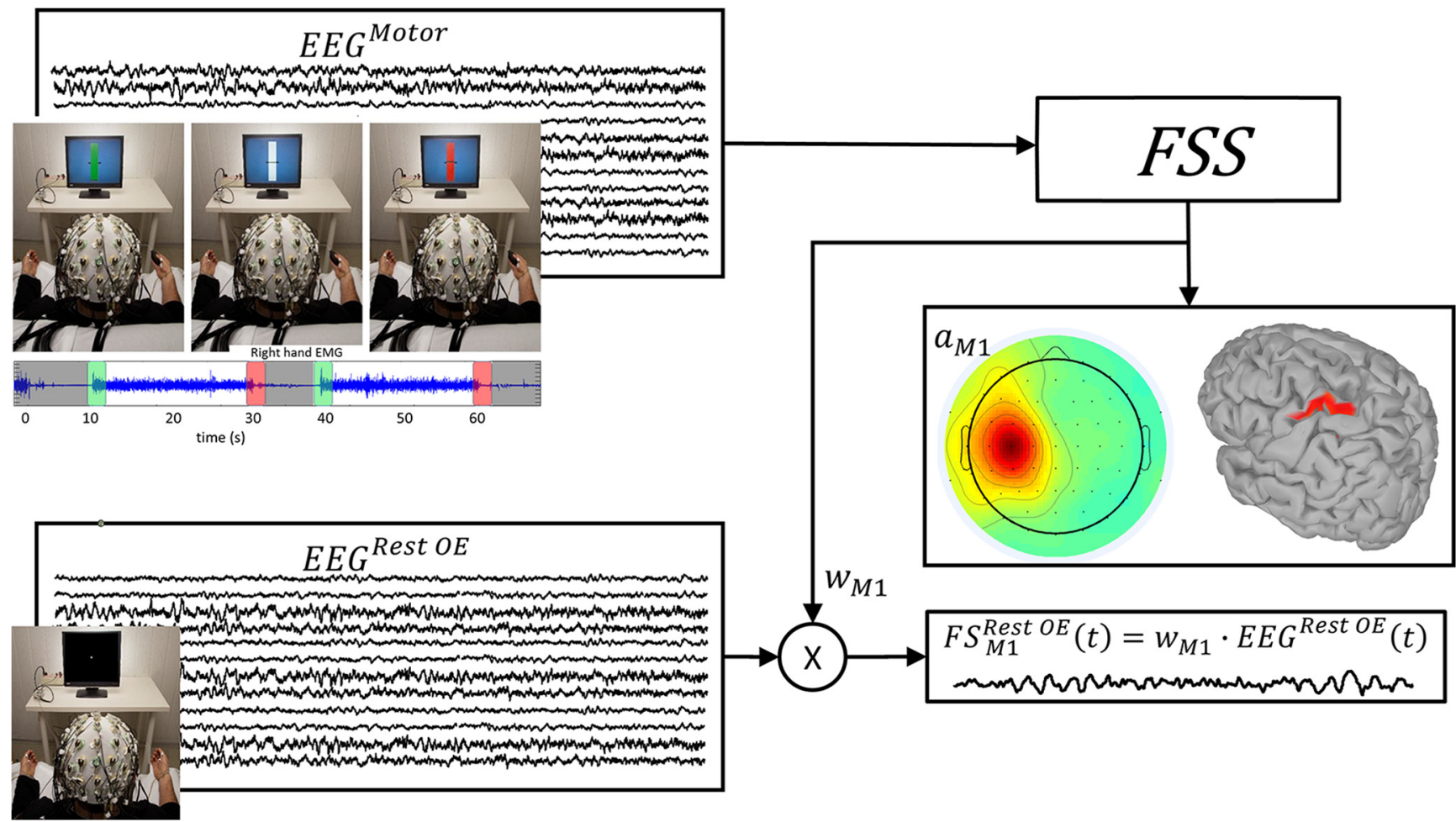

Figure 1. Obtaining individual $M 1$ neurodynamics at rest. A schematic depiction of how $F S S$ obtains the functional source within the primary motor cortex devoted to the right hand (FS $\left.\mathrm{M}_{1}\right)$ for each subject while he/she was resting with open eyes (Rest OE). FSS received the EEG data recorded during an isometric handgrip executed by the right hand (motor condition, white time intervals in top left inset) as input and provided the $\mathrm{a}_{M 1}$ as an outcome (which, similar to $\omega_{M 1}$, is a 64-dimension vector). The $\mathrm{FS}_{\mathrm{M} 1}$ scalp topography derived from $\mathrm{a}_{\mathrm{M} 1}$ is shown for the grand average across 16 subjects, together with its localization obtained by solving the inverse problem using sLORETA (Pascual-Marqui, 2002) within the Brainstorm standard head model (http://neuroimage.usc.edu/ brainstorm; middle right inset). $\mathrm{FS}_{\mathrm{Ml}}^{\mathrm{Rest}} \mathrm{OE}$ (bottom right inset) is obtained through $\omega_{F S}$ multiplied with the EEG recording at rest with open eyes (bottom left inset).

The aim of the present study was to provide evidence that a transcranial electric stimulation (tES), characterized by a current time course that mimics the endogenous electrical activity of the target neuronal pools, effectively changes the pool's excitability.

We assessed the individual neurodynamics of the pyramidal neurons of the hand primary motor cortex $\left(\mathrm{Ml}_{\text {hand }}^{\text {Pyr }}\right)$ of each of our subjects by applying Functional Source Separation (FSS) (Tecchio et al., 2007; Porcaro and Tecchio, 2014) to their EEGs. We set up a stimulation that mimics the individual subject's $\mathrm{M1}_{\text {hand }}^{\text {Pyr }}$ neurodynamics $\left(\mathrm{FS}^{\mathrm{Ml}}(t)\right.$, in the $1-250 \mathrm{~Hz}$ range), to be delivered through a standard electric stimulator and electrodes. We called our protocol "transcranial individual neurodynamics stimulation" (tIDS). We tested the efficacy of tIDS by using a wellestablished online paradigm (Feurra et al., 2011; Tecchio et al., 2013; Cancelli et al., 2015a, b). After testing the neuromodulation efficacy of tIDS, we compared it with the one offered by the transcranial alternating current stimulation (tACS) at $20 \mathrm{~Hz}$, which is considered the most effective noninvasive method for online enhancing the excitability of the corticospinal tract (Feurra et al., 2011). In addition to the standard sham control condition and tACS, we included in our protocol the transcranial random noise stimulation (tRNS), widely presented in the literature. Furthermore, as a fifth condition, we used tIDS with a lower current intensity. Indeed, it has been observed that, at "sufficiently low" current intensities, timevarying tES produces inhibition, rather than enhancement, of cortical excitability (Moliadze et al., 2012; Cancelli et al., 2015b).

\section{Materials and Methods}

The study was approved by the Ethics Committee of San Giovanni Calibita-Fatebenefratelli Hospital.

Our paradigm consisted of the following two parts: the EEG session to set up tIDS and the tES/transcranial magnetic stimulation (TMS) session to test tIDS efficacy. The first part of the paradigm requires the EEG to be integrated with FSS to obtain the neurodynamics of $\mathrm{M} 1_{\text {hand }}^{\text {Pyr }}$, which is then mimicked by tIDS. In the second part of the paradigm, the tES/TMS session assesses the excitability of the hand corticospinal tract from the motor-evoked potentials (MEPs) collected during tES on and off for each stimulation (tIDS, tACS, tRNS, sham, and tIDS $_{\text {low }}$ ).

\section{Participants}

Eighteen right-handed healthy subjects with normal neurological examination results and medical histories were included in the study after providing signed informed consent. None of the volunteers had taken psychoactive drugs for 6 months before the study. All subjects received $€ 15$ for their participation.

\section{Obtaining individual M1 neurodynamics from EEG recordings}

To obtain the individual $\mathrm{M}_{\text {hand }}^{\text {Pyr }}$ neurodynamics, each subject underwent an EEG recording (64-channel actiCHamp System; Brain Products) and a surface EMG of the right opponens pollicis (OP) muscle. EEGs and EMGs were sampled at $5 \mathrm{kHz}$ (presampling analogical bandpass filtering 0.1-2000 Hz) and stored for offline processing. By using FSS (Tecchio et al., 2007; Porcaro and Tecchio, 2014), we identified the neurodynamics of M1's pyramidal neurons devoted to the control of the contralateral hand, and to the thumb in particular, at rest (Porcaro et al., 2008).

Here, we provide a full description of the experimental setting and FSS procedure, which previously provided the ability to identify the activity of M1's pyramidal neurons (Porcaro et al., 2008; Tecchio et al., 2008b; Betti et al., 2009; Pittaccio et al., 2011; Melgari et al., 2013).

\section{Experimental EEG paradigm}

EEGs were recorded while the subject executed an isometric handgrip against the resistance of a semicompliant air bulb (Fig. 1, top left), connected to a digital board that recorded the exerted pressure and sent a 
visual feedback of that pressure to a computer screen placed in front of the subject (Interactive Pressure Sensor; Consiglio Nazionale delle Ricerche in-house developed Arduino-based device). The motor condition was divided into two parts. First, we asked the subject to squeeze the bulb as strongly as possible to assess the subject's right handgrip maximum voluntary contraction. We then let him/her relax for at least $2 \mathrm{~min}$ to avoid fatigue side effects. After resting, the subject was shown a vertical rectangle-shaped white gauge over a light blue background at the center of the computer screen (Fig. 1, top left). Two black segments on either side of the gauge indicated a force level equal to $5 \%$ of the subject's maximum voluntary contraction. A moving black horizontal bar within the gauge provided visual feedback of the pressure exerted on the bulb. At this point, the subject was asked to squeeze the bulb and maintain an isometric contraction sufficient to keep the central moving bar leveled with the two fixed lateral bars. The task was started with a "go" and ended with a "stop" sign on the screen. The contraction periods lasted $20 \mathrm{~s}$, separated by 10 s periods of rest.

EEGs were recorded during all parts of the motor condition. In addition, EEGs were also collected for $3 \mathrm{~min}$ while the subject relaxed and looked at a fixation point at the center of the screen (Fig. 1, bottom right).

\section{M1 identification by FSS-equipped EEGs}

Details of the FSS algorithm were published in previous papers (for review, see Tecchio et al., 2007; Porcaro and Tecchio, 2014). In summary, FSS follows the classic independent component analysis (ICA) assumption that the EEG traces recorded by $m$ electrodes $\mathbf{X}(t)=\left[\mathrm{x}_{1}(t), \ldots, \mathrm{x}_{\mathrm{m}}(t)\right]$ are a linear combination of signals generated by $n$ unknown neuronal sources $\mathbf{S}(t)=\left[\mathrm{s}_{1}(t), \ldots, \mathrm{s}_{\mathrm{n}}(t)\right]$, which are mixed by an unknown mixing matrix $\mathbf{A}$ as follows:

$$
X(t)=A S(t)
$$

ICA's aim is to find A and consequently $\mathbf{S}(t)$ starting from the available $\mathbf{X}(t)$, without making any particular assumption other than statistical independence of the unknown sources. To obtain the sources $\mathbf{S}(t)$, the inverse matrix $\mathbf{W}=\mathbf{A}^{-1}$ must be estimated. Therefore,

$$
\boldsymbol{W X}(t)=W A S(t) \Rightarrow S(t)=W X(t)
$$

FSS adds one or more functional constraints to the standard ICA assumptions. The FSS additional assumption is that known functional characteristics of the areas under study can be included in the ICA computation. Thus, FFS identifies the source that maximizes a functional constraint $R$ believed a priori to be associated with the neuronal pool to be identified. FSS starts from the original EEG data $\mathbf{X}(t)$ and returns one functional source $\mathrm{FS}(t)$ at a time as follows:

$$
F S(t)=a_{F S}^{-1} \boldsymbol{X}(t)=w_{F S} \boldsymbol{X}(t)
$$

where $a_{F S}^{-1}$ is the mixing time-invariant vector and $\omega_{F S}$ is the separation vector. Because FSS searches for a single source solution, $\mathrm{FS}(t)$ is a time vector.

To identify the "hand" region of the primary motor area (named $\mathrm{FS}_{\mathrm{M1}}$ ), FSS used the coupling of cortical and muscular rhythmic oscillations (cortico-muscular coherence $[\mathrm{CMC}]$ ) in the $\beta$ band during the isometric handgrip (motor) task (Porcaro et al., 2008). The corresponding functional constraint is as follows:

$$
R_{F S_{M 1}}=\sum_{\omega_{\max }-\Delta_{1} \omega_{\max }}^{\omega_{\max }-\Delta_{2} \omega_{\max }} \operatorname{Coh}(\omega)
$$

where Coh is the coherence function. For each $\omega$, Coh is the amplitude of the cross-spectrum between the $\mathrm{FS}_{\mathrm{M} 1}$ source signal and the nonrectified EMG, normalized by the root mean square of the power spectral densities of these two signals. $\Delta_{1} \omega_{\max }\left(\Delta_{2} \omega_{\max }\right)$ is the frequency point corresponding to a coherence amplitude that is $50 \%$ of the maximum value within the range from 13.5 to $33 \mathrm{~Hz}$.

When $R_{F S_{M 1}}$ is maximized, FSS can estimate $\alpha_{M 1}$ and be used to obtain $F S_{M 1}^{M O T O R}(t)$ according to the following formula:

$$
F S_{M 1}^{\text {MOTOR }}(t)=a_{M 1}^{-1} X^{\text {MOTOR }}(t)=w_{M 1} X^{\text {MOTOR }}(t)
$$

The significance of the $\mathrm{CMC}$ value was estimated according to the following (Halliday et al., 1995): $y 1-0.05^{\frac{1}{L-1}}$, where $L$ is the number of averaged windows during the isometric contraction $(\sim 300$, resulting in a limit of 0.01).

Once $\mathrm{a}_{\mathrm{M} 1}$ and $\omega_{M 1}(1 \times 64$ values $)$ were estimated, M1's cortical activity during the rest condition with open eyes (Rest OE) was obtained starting from EEG rest recordings, EEG ${ }^{\text {Rest } \mathrm{OE}}(t)$ (Cottone et al., 2017) (Fig. 1) as follows:

$$
F S_{M 1}^{\text {Rest } O E}(t)=w_{M 1} E E G^{\text {Rest } O E}(t)
$$

\section{tIDS replaying M1 neurodynamics at rest}

tIDS delivers a time-varying current by replaying $F S_{M 1}^{\text {Rest }} O E(t)$, which is the individual M1 neurodynamics at rest, through two standard tES electrodes (see tES below).

Notably, we found a relevant intersubject variability of $F S_{M 1}^{\text {Rest }}{ }^{O E}(t)$ frequency components (Fig. 2).

\section{tES/TMS study design and sample size estimate}

$t E S$

We used two equal $7 \times 5 \mathrm{~cm}^{2}$ rectangle electrodes positioned above $\mathrm{C} 3$ and $\mathrm{Oz}$. All waveforms were produced by a function generator $(2 \mathrm{MHz}$ USB PC Function Generator PCGU1000, Velleman Instruments) connected to a current stimulator (STMISOLA linear isolated stimulator, Biopac System).

We provided, in a random order across subjects, the following four different transcranial current waveforms and sham (sampling rate 500 sample per second; Fig. $3 A$ ):

- tACS: a $20 \mathrm{~Hz}$ sinusoidal stimulation.

- tRNS: a random level of current generated for every sample in the $1-250 \mathrm{~Hz}$ range. The probability density was a bell-shaped function. Consequently, in the frequency spectrum, all frequency bins had similar coefficients (Gaussian white noise).

- tIDS: a current stimulation replaying the individual M1 neurodynamics as described above.

- $\mathrm{tIDS}_{\text {low }}$ : identical to tIDS but with current density selected to induce inhibition as reported by Moliadze et al. (2012) and Cancelli et al. (2015b).

- Sham: the $6 \mathrm{~s}$ of current ramp at the beginning and end of the 1.5 min period.

All stimulations ramped up and down over the first and last $6 \mathrm{~s}$ of stimulation. No DC offset was present in either stimulation.

As our aim was to set the IIDS efficacy and compare it with the efficacy of the diverse $\mathrm{tES}$, we equated the current intensities of the compared conditions (tACS, tIDS, and tRNS), thus providing the same power in the three conditions. As the current intensity reference, we chose $20 \mathrm{~Hz}$ tACS at $1 \mathrm{~mA}$ peak-to-peak, which has been shown to enhance the corticospinal excitability (Feurra et al., 2011, 2013; Cancelli et al., 2015a, b). Consequently, we adjusted the tIDS and tRNS current intensities to deliver the same power of tACS. In particular, the multiplicative coefficients for the $1.5 \mathrm{~min}$ stimulations were as follows:

$$
\begin{gathered}
\rho_{t I D S}=\sqrt{\frac{P_{t A C S 1 \mathrm{~mA} p p}}{P_{t I D S}}} \\
\rho_{t R N S}=\sqrt{\frac{P_{t A C S 1 \mathrm{~mA} p p}}{P_{t R N S}}}
\end{gathered}
$$

where $P_{t A C S ~ 1 m A ~ p p}$ is the power of tACS at an intensity of $1 \mathrm{~mA}$ peak-topeak, and $P_{t I D S}$ and $P_{t R N S}$ are the power of tIDS and tRNS with the peak posed equal to 1 , respectively. The factor $P_{t I D S}$ for tIDS was similar across subjects, with a mean and corresponding SD of $1.065 \pm 0.004$. Regarding tIDS $_{\text {low }}$, we set the adjustment coefficient to make the delivered power equal to that of the $0.85 \mathrm{~mA}$ peak-to-peak $20 \mathrm{~Hz}$ sinusoidal signal previously used to inhibit M1 excitability as follows (Moliadze et al., 2012; Cancelli et al., 2015b): 

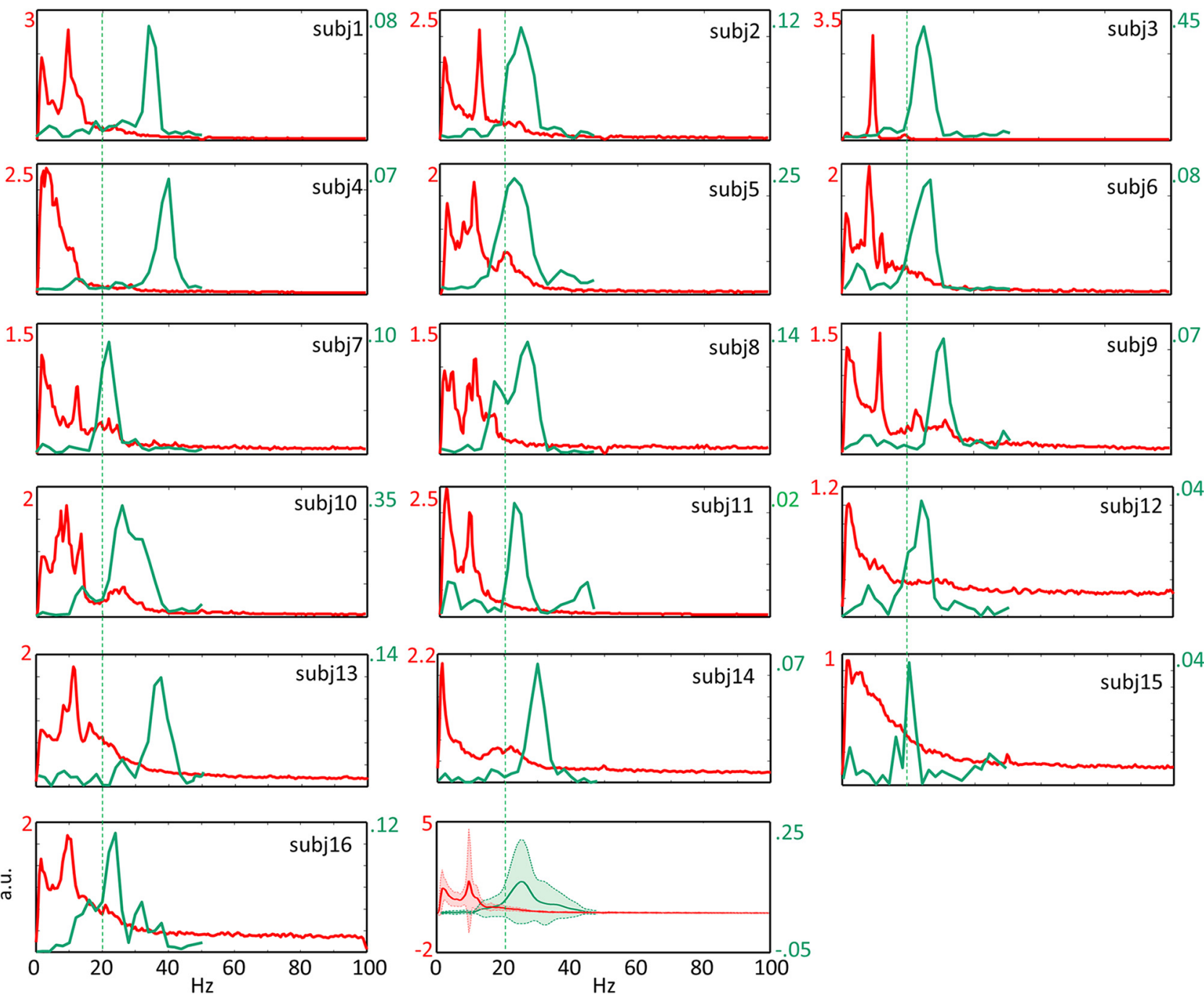

Figure 2. Individual $\mathrm{FS}_{\mathrm{M} 1}$ power spectral densities and $\mathrm{FS}_{\mathrm{M} 1}$ coherence with the muscle. Red represents the power spectral density (PSD) of the $F S_{\mathrm{M} 1}$ in the 3 min period of a resting state with open eyes for each subject (Figs. 3-5 are color-coded similarly). PSD values are calculated equaling the peak of FS $\mathrm{M}_{\mathrm{M} 1}(t)$ to 1 . Green represents the coherence between $\mathrm{FS}_{\mathrm{M} 1}$ and the $0 \mathrm{P}^{\prime} \mathrm{EMG}(\mathrm{CMC})$ during the $\sim 3$ min of 20 s periods of isometric contractions. Vertical dashed line indicates $20 \mathrm{~Hz}$. The last plot represents the means and SDs across 16 subjects. PSD and CMC scales are shown in respective colors.

$$
\rho_{\text {tIDS }}=\sqrt{\frac{P_{\text {tACS } 0.85 \mathrm{mApp}}}{P_{\text {tIDS }}}}
$$

\section{TMS probing protocol}

To probe tIDS efficacy and differential neuromodulation effects depending on the diverse tES, we executed a paradigm that tests the neuromodulation efficacy during stimulation (Feurra et al., 2011; Tecchio et al., 2013; Cancelli et al., 2015a, b). We performed single-pulse TMS through a standard focal coil (HP $90 \mathrm{~mm}$ Coil 9784-00) connected to a SuperRapid module (Magstim), with the coil placed on the tES rolandic electrode. We induced the TMS-MEPs in the OP of the right hand. We recorded MEPs with surface electrodes in a belly-tendon montage ( $2.5 \mathrm{~cm}$ apart). The hot spot of the right OP muscle was identified (TMS coil positioned above the tES electrode). Thereafter, we digitized the coil position and monitored it throughout the session with the SofTaxic neuronavigator. For each subject sitting relaxed with open eyes, the TMS was applied at an intensity adjusted to produce OP MEP amplitudes of $\sim 0.5-1 \mathrm{mV}$ in basal conditions (i.e., TMS applied through the tES electrode, with tES off). After 1.5 min of rest (TMS off - tES off), TMS was switched on to collect the baseline MEPs (TMS on tES off) for $1.5 \mathrm{~min}$. Eventually, the tES was switched on as well for $1.5 \mathrm{~min}$ (TMS on - tES on). We always waited at least $1.5 \mathrm{~min}$, in complete rest (no TMS), before collecting the baseline of the new tES. Because TMS stimuli were elicited with an interstimulus interval that changed randomly between
$4.5 \mathrm{~s}$ and $5.5 \mathrm{~s}$, we collected $\sim 18$ MEPs for the baseline and with the tES on. This was repeated for each of the five tESs in a random order (Fig. $3 B$ ). In this way, we enriched the protocol introduced by Feurra et al. (2011) by collecting a baseline (TMS on - tES off) in the interval just before each tES was switched on (Cancelli et al., 2015a, b). This means that each tES had the MEP baseline collected for each condition (for $1.5 \mathrm{~min}$ before tES stimulation) instead of referring all tES conditions to a single baseline collected at the beginning of the experiment. In the setup of Feurra et al. (2011), increasingly longer times separated the tES condition of interest from the baseline, and the strength of the comparison among different $\mathrm{tES}$ is guaranteed by the random order of different tESs across subjects (Feurra et al., 2011).

We asked all participants to report immediately whether any stimulation was provoking a special sensation. Indeed, the stimulations lasting $1.5 \mathrm{~min}$, alternated with $3 \mathrm{~min}$ of relaxation, did not allow for the filling in of a form during the protocol. Furthermore, at the end of each tES delivery period, we asked whether the last stimulation had been real or sham.

\section{Statistical analysis}

After a logarithmic transformation of single MEP amplitudes in single subjects, we confirmed that the Gaussian distribution provided a good fit by performing the Shapiro-Wilk test. Thereafter, we computed the normalized MEPs as described, and we submitted them (dependent variable) to the following analyses. 


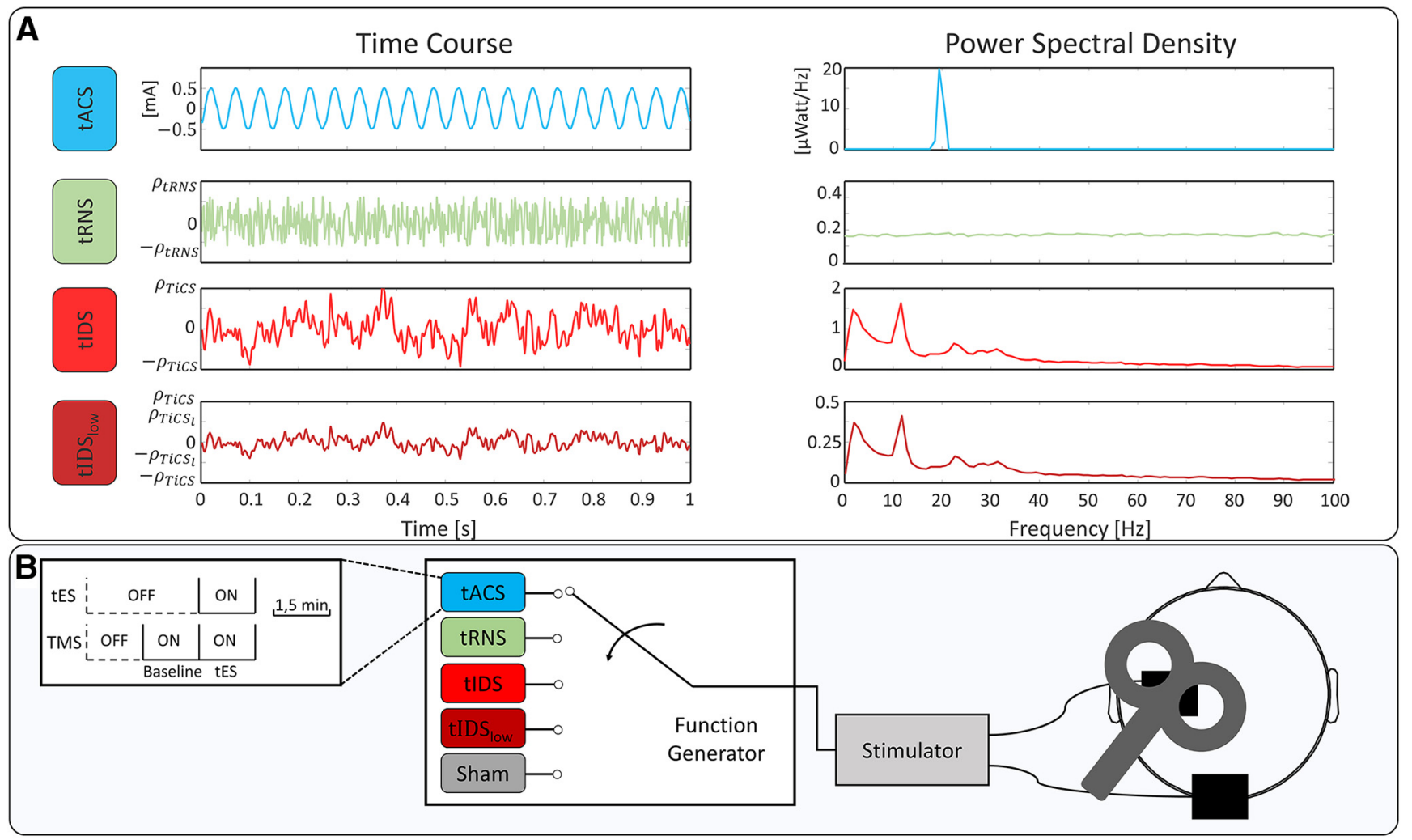

Figure 3. Experimental setup for testing tIDS neuromodulation efficacy. $\boldsymbol{A}$, In a representative subject, the time course (left, $\mathrm{mA}$ ) and power spectral density (right, $\mu$ Watt/Hz) of the four tESs

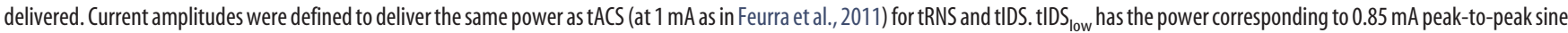
(20 Hz tACS as in Cancelli et al., 2015b). PSD scale is $1 / 4$ of the tIDS scale. B, Representation of the tES/TMS session: each of the 5 tES stimulations (tACS, tRNS, tIDS, tIDS low' and sham, delivered in random order for each subject) lasted 1.5 min and was alternated with intervals of 3 min rest. tESs were delivered through two equal $7 \times 5 \mathrm{~cm}^{2}$ rectangle electrodes positioned above $C 3$ and $0 z$ and the TMS coil positioned above C3. For each tES, we delivered TMS during the 1.5 min while the tES was on and in the previous 1.5 min with ES off, providing the baseline MEP values for the following stimulation.

The first analysis aimed at evaluating whether tIDS enhanced MEP amplitude in on versus off stimulation. The ANOVA included Stimulation Switch (On, Off) as a fixed factor and Subjects as a random factor.

To assess the second aim, we applied the full model ANOVA with Subjects as a random factor and $t E S$ (tACS, tRNS, tIDS, tIDS $_{\text {low }}$, and sham) and the Stimulation Switch (On, Off) as fixed factors. When the tES * Stimulation Switch interaction effect appeared, we executed post hoc comparisons. In particular, we evaluated whether the values when stimulation was on differed from those when the stimulation was off and from sham. We also evaluated whether the baseline MEPs changed throughout the experiment using the same analysis design (omitting the Stimulation switch factor), applied to the baseline $\log (\mathrm{MEP})$ values.

Furthermore, to strengthen information about the individual M1 excitability enhancement, we compared in each single subject all the repetitions at baseline and tES (Tecchio et al., 2008a) by using a two-tailed independent sample $t$ test for those stimulations, which induced an enhancement in the population as evidenced by the above analysis. Finally, we compared these individual $t$ values for tIDS and tACS by means of a paired-sample $t$ test.

We executed the statistical analysis using SPSS (IBM).

\section{Results}

FSS-equipped EEG for M1 identification

We excluded 2 subjects who did not display CMC above the confidence limit of $95 \%$. We successfully identified the M1 neurodynamics for each of the other 16 subjects (10 females, 6 males; age range: $19-51$ years, mean age: $30.1 \pm 8.9$ years).

\section{tES/TMS paradigm for testing tIDS efficacy}

No participant reported any specific sensation during the stimulations. As shown in Table 1, more than half of the people did not perceive that a stimulation occurred in any $\mathrm{tES}$. In particular,
Table 1. Personal sensation during tES ${ }^{a}$

\begin{tabular}{|c|c|c|c|c|c|}
\hline & \multicolumn{5}{|c|}{ Stimulation } \\
\hline & tACS & tRNS & tIDS & tIDS $_{\text {low }}$ & Sham \\
\hline Real & 6 & 2 & 3 & 5 & 3 \\
\hline Sham & 10 & 14 & 13 & 11 & 13 \\
\hline
\end{tabular}

${ }^{a}$ Number of people answering " $\mathrm{R}$ " or " $\mathrm{S}$ " immediately after stimulation completion.

fewer people perceived a stimulation during tIDS than during tACS and IDS $_{\text {low: }}$.

The mean resting motor threshold across subjects was $58.5 \pm$ $8.1 \%$ of the maximal TMS stimulator output, and the stimulation intensity used during the experiment was, on average, $70.8 \pm$ $10.1 \%$. We submitted the amplitudes of all collected MEPs to the analysis, without applying any selection. In each condition, we collected a median of 17 MEPs (total matrix cell number: 2609), with no difference in the number of stimuli across conditions ( $p>0.400$ consistently).

\section{Complete experiment ( 5 conditions, 11 subjects)}

No change in baseline levels occurred, as indicated by $t E S$ Baseline $\left(F_{(4,40.938)}=0.867 ; p=0.492\right.$; Table 2; Figure $\left.4 A\right)$. The absence of relevant variations of baseline MEPs showed that the preceding tES induced negligible aftereffects, at least waiting $1.5 \mathrm{~min}$ after the stimulation. The stability of the M1 excitability after the other tES with no relevant carryover effects strengthens the suitability of this online probing protocol in testing specific features of tES.

The ANOVA applied to the normalized MEPs devoted to assess the tIDS ability to enhance MEP amplitudes (first aim) indi- 
Table 2. Baseline MEP amplitudes ${ }^{a}$

\begin{tabular}{lrll}
\hline & Mean & $95 \%$ lower limit & $95 \%$ higher limit \\
\hline tACS & 922.9 & 562.2 & 1283.5 \\
tRNS & 967.8 & 656.1 & 1279.7 \\
tIDS & 861.6 & 393.4 & 1329.8 \\
tIDS & 1141.9 & 797.6 & 1486.3 \\
Sham & 817.2 & 249.0 & 1385.5 \\
\hline
\end{tabular}

${ }^{a}$ Baseline values (i.e., MEP amplitude values when tESs were off). Values are exponential back-transformation of the means of logarithmic transformed individual values.

cated a clear tIDS efficacy (Stimulation Switch effect $F_{(1,11.663)}=$ 15.429, $p=0.002)$, without any difference across subjects (Stimulation Switch ${ }^{*}$ Subject effect $\left.F_{(10,306)}=0.891, p=0.542\right)$.

The full model ANOVA showed that the neuromodulation efficacy changed with the dynamics of the delivered current $\left(t E S\right.$ * Stimulation Switch interaction effect $\left(F_{(4,46.346)}=4.200 ; p=\right.$ 0.006; Figure $4 B)$. Notably, the triple interaction $t E S{ }^{*}$ Stimulation Switch ${ }^{*}$ Subject $(p=0.772)$ indicated a highly stable pattern of the five tES neuromodulation effects across subjects. Post hoc analysis showed that only tIDS differed between on and off $(p<0.001$, Bonferroni corrected; Fig. $4 B$ ) and a slight difference was found for tACS $(p=0.051)$. The other tRNS, tIDSlow, and sham showed a significance of $p>0.500$ consistently. In addition, only tIDS differed from sham (tES on condition $p=0.003$ ), whereas tACS did not $(p=$ 0.313 ). Furthermore, tRNS and tIDSlow both showed a significance of $p>0.600$ (Fig. $4 B$ ). The mean MEP amplitude increase was $32 \%$ of the baseline during tIDS and 16\% during tACS.

\section{Experiment for the main effects ( 3 conditions, 16 subjects)}

tIDS induced a clear enhancement of MEP amplitudes (Stimulation Switch effect $\left.F_{(1,16.864)}=24.330, p<0.001\right)$, with no relevant difference across subjects (Stimulation Switch ${ }^{*}$ Subject effect, $\left.F_{(15,479)}=1.106, p=0.347\right)$.

Executing the full model in the 16 subjects with the three tIDS, $20 \mathrm{~Hz}$ tACS, and sham conditions, no change in baseline levels occurred $\left(t E S\right.$ Baseline $F_{(2,30.677)}=1.784 ; p=0.185$; Figure $4 A$ ). The dynamics of the delivered current had a strong effect on neuromodulation efficacy, indicated by the $t E S{ }^{*}$ Stimulation Switch interaction effect $\left(F_{(2,33,330)}=7.735 ; p=0.002\right.$; Figure $4 B)$. The triple interaction $t E S{ }^{*}$ Stimulation Switch ${ }^{\star}$ Subject effect was absent $(p=0.496)$. Post hoc analysis showed that only tIDS differed between the on and off states $(p<0.001$, Bonferroni corrected; Fig. $4 B$ ), and a slight difference was found for tACS $(p=0.096)$. In addition, only tIDS differed from sham (tES on condition, $p<0.001)$, whereas tACS did not $(p=0.458)$. Both tACS and tIDS differed from the $100 \%$ value (one-sample $t$ test, $p=0.015$ and $p<0.001$, respectively). Considering the 16 people, tIDS differed from tACS ( $p<0.001$; Fig. $4 B$ ).

On an individual basis, we observed that the MEP amplitude increase induced by tIDS was higher than that induced by tACS in 13 of 16 subjects (Fig. $5 A, C$ ). When we compared the $t$ values of the independent paired-sample $t$ test that compared off and on MEPs for tIDS to tACS, we found that tIDS was more effective than tACS $(p=0.001)$. The MEP potentiation in single subjects evaluated by an independent-sample $t$ test considering all MEPs repetitions during the tES on versus tES off at a significance threshold of $p=0.100$ showed potentiation in no subjects during tACS and in 8 of 16 subjects during tIDS (Fig. 5A). The mean MEP amplitude increase was $34 \%$ of the baseline during tIDS and 13\% during tACS (Fig. 5C).

\section{Discussion}

The main finding of our work was that a tES with a current time course, which replays the ongoing electrical activity of the target area
(tIDS), produces effective neuromodulation. In particular, tIDS produces an effective potentiation in 8 of 16 subjects. Furthermore, tIDS produced more effective neuromodulation than other available noninvasive transcranial stimulation protocols (20 Hz tACS).

\section{Model of tIDS mechanism of action}

Here, we introduced a model that hypothesized the mechanisms causing corticospinal neurons to enhance excitability during tIDS. We considered a set of nodes and their connections as a neural network (nn). In particular, in our case, the node is the hand section of pyramidal neurons of the primary motor area. In our model, each node of an nn develops "typical" dynamics of neuronal activity during its lifespan. In agreement with this model, we observed that $\mathrm{M}_{\text {hand }}^{\text {Pyr }}$ has "its own" dynamics (Cottone et al., 2017). An nn implements a function via the communication among its nodes (i.e., the nodes of an nn share a "common language"). Once a signal arrives to a node ("word-in"), it automatically answers with a "word-out" determined by a fixed "word-in $\rightarrow$ word-out" correspondence until plasticity modifies that correspondence. In other words, in an nn, each node cannot stay silent; it necessarily produces the "word-out" once the "word-in" arrives. Our hypothesis in this model was that, using tIDS, we "speak" to M1 neurons with their "typical language" (i.e., the one they use to produce their physiological output). In our hypothesis, the neuronal pools change their probability to activate (i.e., to send their message to other neuronal pools when a signal similar to the one they typically send impinges their membranes). In this model, M1 neurons communicate with connected neurons (motor neurons or other cerebral areas) by patterns, which express at typical "rates" that are only roughly represented by sinusoidal packages (Ainsworth et al., 2012; Histed and Maunsell, 2014). To establish functional communication relevant for behavior, neuronal nodes convey signals with a temporal structure that is critical to the ways in which signals are routed, combined, and coordinated (Ecker et al., 2010; Renart et al., 2010; Kopell et al., 2014). In particular, two pieces of evidence, in addition to tIDS being more effective than tACS, support this model. Beta-tACS changes the neuronal excitability, whereas alpha-tACS does not (Feurra et al., 2011). If the tESs were entering some "resonance" with the dominant frequency of local activity, we would predict that alpha- would be more effective than beta-tACS because the local spectra have higher alpha than beta power in all subjects (see also the Fig. 2 in our specific case). Moreover, tES would cause a reduction of excitability because higher power of the ongoing activity corresponds to a lower excitability (Engel et al., 2001; Jensen et al., 2014; Thut, 2014; Haegens et al., 2015; de Pesters et al., 2016). Instead, the lack of alpha-tACS effects and the evidence that betatACS enhances M1 excitability indicated that tES is effective when the stimulation matches the language that the target node uses to fulfill its functional role. Indeed, M1 uses beta synchronizations to communicate with muscles. Fröhlich and McCormick (2010) observed that either endogenous or exogenous naturalistic sinusoidal stimulation could "entrain" neocortical network activity (an in vitro study). Furthermore, Gal and Marom (2013) demonstrated that the electrical activity of single cortical neurons of rats in vitro shows scale-free fluctuations, which can be entrained by delivering natural-like, scale-free inputs. In our study, we enhanced the efficacy of noninvasive cortical pool neuromodulation by using their own dynamics (Cottone et al., 2017).

In the absence of any gold standard, we compared the neuromodulation efficacy of our novel tES with the one so far known to be the most effective in inducing an excitability enhancement of the corticospinal tract during current delivery (i.e., tACS at 20 
A

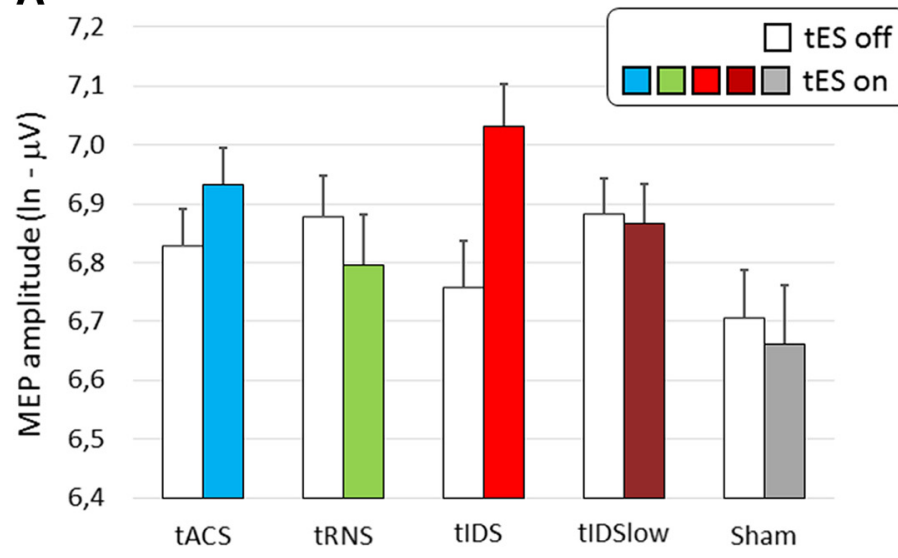

B

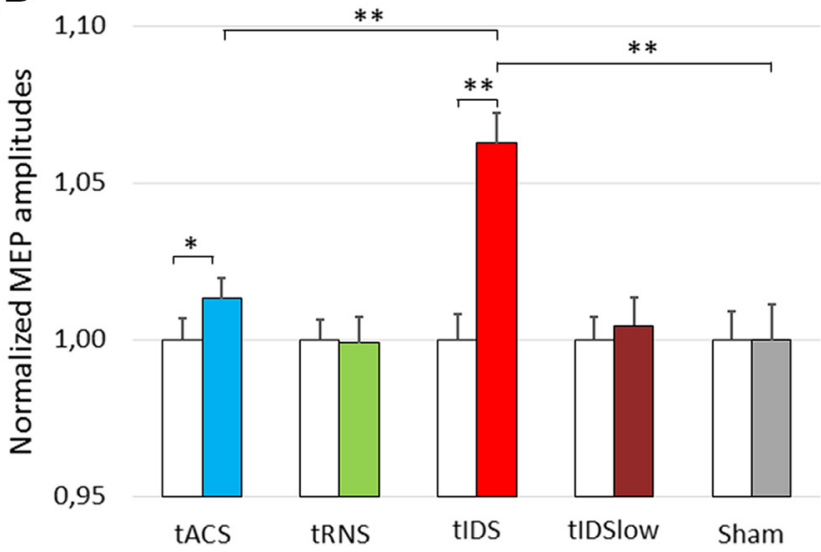

Figure 4. tES neuromodulation efficacy: mean across subjects. Representation of the mean for 16 subjects for tACS, tIDS, and sham, and the mean for 11 subjects for tRNS and tIDS Iow $_{\text {. }}$, Means of the logarithmic transformed amplitudes of the TMS MEPs with tES off (while bars) and tES on (color codes in figure for the four current modulations and sham). $\boldsymbol{B}$, Means across subjects of the normalized MEPs during tES on and tES off (see Materials and Methods): each MEP divided for the mean in baseline; thus, the tES off mean of the normalized MEPs is always 1 . Vertical bars represent SEs. Differing values between the contrasted conditions: ${ }^{* *} p<0.010 ;{ }^{*} p<0.100$.
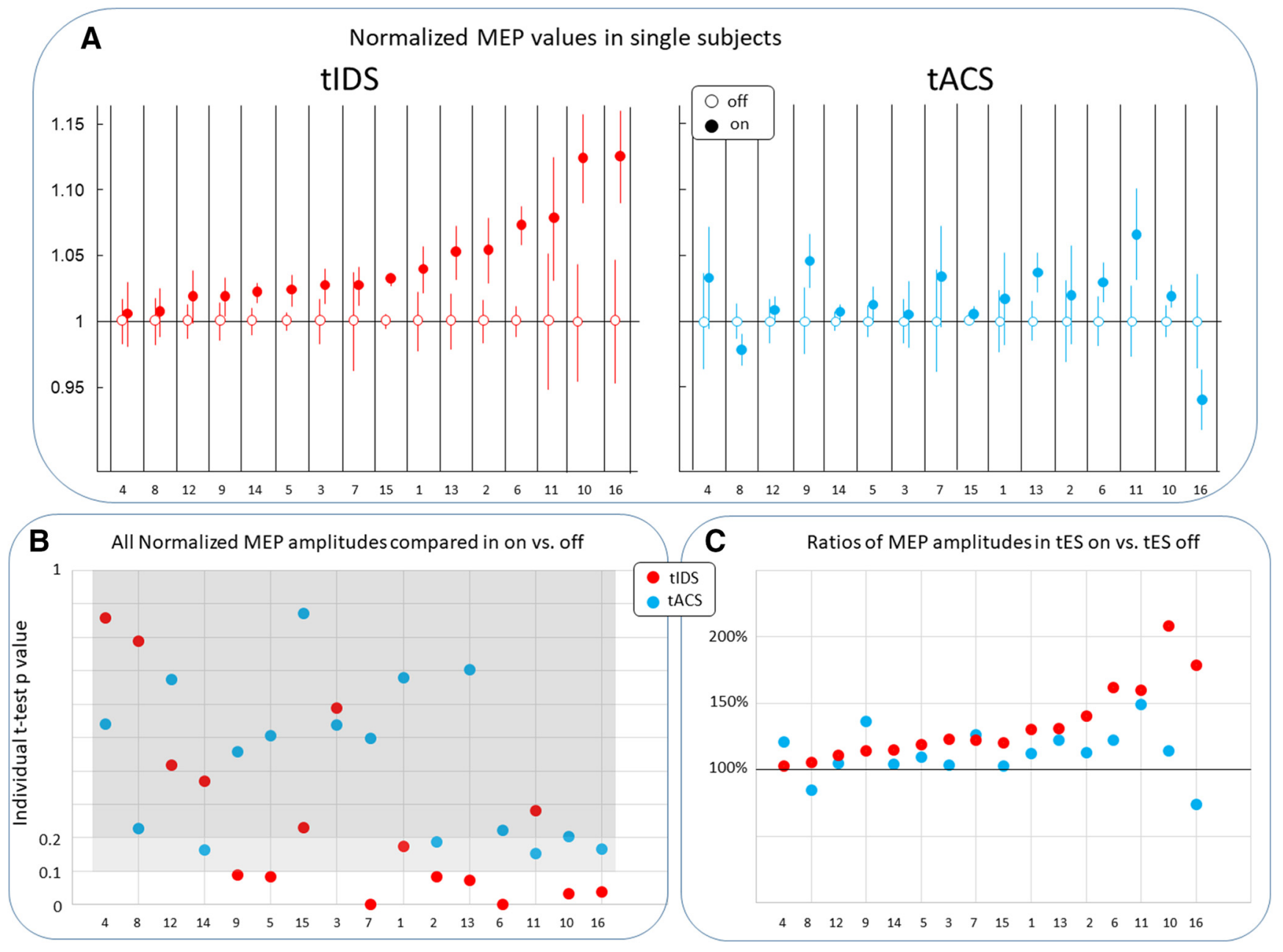

Figure 5. Efficacious neuromodulations: individual effects. In each individual subject, ordered for tIDS response. $A$, Individual means and SE of normalized MEP for tACS and tIDS. $\boldsymbol{B}, p$ values of the individual comparison of all repetitions in off and on tES, for tACS (light blue), and tIDS (red) by independent-sample two-tailed $t$ test. tACS did not induce an effect $<0.100$ of the significance threshold (white area) in any of the subjects, whereas tIDS induced this effect in 8 of 16 subjects. Lighter gray area represents $0.100<p<0.200$. Darker gray area indicates $p>0.200$. C, The percentage changes of MEP during tES on with respect to tES off for the two tACS and tIDS. Black horizontal line indicates no change (i.e., 100\%). It is noteworthy that, in the 5 subjects with tACS at significance $<0.200$ (Subjects 2, 10, 11, 14, and 16), tIDS induced a greater neuromodulation effect than tACS (significantly more in Subjects 2, 10, and 16). 
$\mathrm{Hz}$ ) proposed by Feurra et al. (2011). These authors considered the fact that different brain modules mainly oscillate at specific frequencies organized in a topographic manner and posed the working hypothesis that the response to a tACS depends on the modulation frequency. In particular, they showed that the corticospinal excitability increased only when the frequency of the tACS was $20 \mathrm{~Hz}$ (beta range), whereas other frequencies, such as $5 \mathrm{~Hz}$ (delta), $10 \mathrm{~Hz}$ (alpha), and $40 \mathrm{~Hz}$ (gamma), showed no impact on the MEP amplitude. Here, we observed that tACS was less effective than tIDS at the group and at the individual level. Exploiting the newly recruited 5 subject group, we asked whether the personalized tACS was more effective than the nonpersonalized $20 \mathrm{~Hz}$ tACS. That is, we compared the neuromodulation efficacy of a nonpersonalized tACS at $20 \mathrm{~Hz}$, with a tACS at the frequency of maximal CMC (see Fig. 2). We compared the effects in the 4 subjects (Subjects 2, 5, 7, and 8; Fig. 2), and the new 5 subjects (Subjects 12-16) with respect to the other 7 subjects (Subjects 1, 3, 4, 6, 9, 10, and 11). We did not reveal a significant difference in the effects of the two stimulations (normalized MEPs in the on condition, tACS at CMC frequency $v s$. tACS at 20 $\left.\mathrm{Hz}, F_{(1,4.932)}=1,805 ; p=0.238\right)$. It can be that the small number of subjects in the between-subject factor shades a possible small effect. We also executed the analysis to estimate the intrasubject effect comparing the nonpersonalized $20 \mathrm{~Hz}$ tACS and personalized CMC tACS in the 5 subjects (Subjects 12-16), and we found that the tES $(20 \mathrm{~Hz}$ tACS, personalized CMC tACS $)$ *Stimulation (On, Off) factor was absent $(p>0.200)$. These findings support that a single sinusoid stimulation poorly induces neural entrainment at the individual level. Notably, tRNS delivering oscillating current in the same frequency range of tIDS did not produce any effect.

Our data strengthen the notion that we can exploit the complex scale-free dynamics of the local activity to influence the effectiveness of neuromodulation of the target neuronal pools.

\section{Neuronal entrainment and tIDS}

Among the noninvasive neuromodulation techniques using transcranial-induced currents, the mechanisms of action of transcranial direct current stimulation are better elucidated (Brunoni et al., 2011) than those using time-varying currents (Fertonani and Miniussi, 2016) (i.e., tACS and tRNS). Often, the term "entrainment" is used when delivering these two tESs. Christiaan Huygens was one of the first to use the term "entrainment" in reference to the dynamics of a complex system of oscillators, where the energy transfer via phasedependent feedback results in a system evolution from an initial condition of "random" phases of oscillations to a final state where the oscillators swing in synchrony. Here, we use "entrainment" as an effective transfer of delivered energy (i.e., an effective change of the state of the neuromodulation target) without any information about whether this latter changed the neural firing rate and/or the number of activated neurons and/or the interneuronal synchrony. Thus, tIDS entrained target activity in single subjects, wheres even tACS, the most effective up to now, never reached the significance threshold in a single subject. Concurrently, we have shown that these dynamics are region-specific (Cottone et al., 2017), suggesting that this opportunity to entrain according to local neuronal activity dynamics also strengthens the capacity to focus stimulation on predetermined cortical districts.

\section{Goodness of FSS in identifying neuronal pool activities}

Our results of maximal neuromodulation efficacy, induced by a tES modulated to replay the neurodynamics of the individual $\mathrm{M} 1_{\text {hand }}^{\text {Pyr }}$ in resting state, confirm the ability of FSS to identify prop- erly the local neuronal activities. Previously, we showed that FSS can discriminate the "hand" motor representation from its somatosensory (S1) counterpart (Porcaro et al., 2008), despite their proximity and their joint implications in virtually any behavioral event. In addition, we discovered that the functional connectivity between S1 and M1 mediates emphatic sharing (Betti et al., 2009). Furthermore, in the study (Pittaccio et al., 2011), we showed that M1 pyramidal neurons do not modify their activity relative to staying still during passive mobilization, whereas S1's ones do. These diverse behaviors in contiguous cortical areas strengthen the reliability of the identification of brain sources via FSS, M1 pyramidal neurons in particular (Tecchio et al., 2007, 2008b; Melgari et al., 2013). Here, our FSS-assisted neuromodulation protocol works as an efficacious technique to entrain the "hand" M1 pyramidal neurons, as tested via the corticospinal excitability.

\section{Is personalization necessary or is local-dependent neuromodulation sufficient?}

We have reported that diverse spectral features (Tecchio et al., 2008b) and complexity measures (Cottone et al., 2017) can differentiate diverse cortical areas according to their respective neuronal activities, independently of the subject's state (i.e., at rest), performing movements or receiving external stimulations. This means that cortical areas, which differ in their neuronal structure and networking characteristics, also display different neuronal electric activities (Tecchio et al., 2008a; Cottone et al., 2017). The existence of such "typical" dynamics of the cortical area poses the question of whether the individual current modulation is more effective than a "universal" one, which is specific to the stimulated cortical area. Future work will compare "typical" dynamics of M1 in producing a relevant neuronal excitability modulation with respect to the individual M1 dynamics as tested here.

\section{Phase of tIDS}

We did not take into account the phase of the $1.5 \mathrm{~min}$ tIDS with respect to the ongoing cortical activity. We believe that this will not have major effects based on the observation of Crochet et al. (2006) that the change in excitability was similar when sorting out the responses depending on the phase of the spontaneous activity.

\section{State-dependent tIDS}

In the present work, we built tIDS using the neurodynamics while the subject was in a resting state. This is consistent with the experimental setting when tIDS delivered while the subject was resting and relaxed. FSS enables the reconstruction of the stimulated region activity in all of the conditions of interest. Thus, by FSS, we can take into account that the efficacy of neuromodulation with a modulated current depends on what the subject is doing or thinking (Feurra et al., 2013). In particular, the networking nature of motor control can lead to neuromodulation applications where the wanted effect on a certain node realizes by properly acting on other network nodes, for example, the contralateral homologous ones (Brinkman et al., 2016). We can, in the future, deliver a tIDS with the neurodynamics of the target area during the task he/she is executing. In relation to the above model of tIDS mechanism of action, we can hypothesize that, even at rest, the tIDS of M1 may be more efficacious if we deliver the neurodynamics of the motor state because we hypothesized that neuromodulation is more effective when it uses the language that the node uses to produce its function.

\section{Inhibiting versus enhancing neuronal excitability}

Previous work has demonstrated that tACS can induce inhibition or enhancement of the neuronal excitability as a function of the 
superficial current density. Here, we investigated the IDS $_{\text {low }}$ effects with the current density, which inhibited the corticospinal excitability via tACS: both with $20 \mathrm{~Hz}$ (Cancelli et al., 2015b) and $140 \mathrm{~Hz}$ (Moliadze et al., 2012), but we found no inhibition. We interpret this result by hypothesizing that the stronger efficacy in enhancing neuronal excitability corresponds to smaller current intensities that inhibit neuronal activity.

In conclusion, we believe that our work provides new insight into how brain networks function; it proposes a model where the nodes of a neural network communicate via a "typical" language. If we can send a signal similar to the node's language, it changes the likelihood of the node activation. Notably, we opened a fruitful path for neuromodulation applications by offering a new potential to personalize interventions. We can build case-specific tIDSs that aim at compensating for specific alterations of the neuronal functional networking expressed in the altered node's neurodynamics.

\section{References}

Ainsworth M, Lee S, Cunningham MO, Traub RD, Kopell NJ, Whittington MA (2012) Rates and rhythms: a synergistic view of frequency and temporal coding in neuronal networks. Neuron 75:572-583. CrossRef Medline

Betti V, Zappasodi F, Rossini PM, Aglioti SM, Tecchio F (2009) Synchronous with your feelings: sensorimotor $\gamma$ band and empathy for pain. J Neurosci 29:12384-12392. CrossRef Medline

Brinkman L, Stolk A, Marshall TR, Esterer S, Sharp P, Dijkerman HC, de Lange FP, Toni I (2016) Independent causal contributions of alpha- and beta-band oscillations during movement selection. J Neurosci 36:87268733. CrossRef Medline

Brunoni AR, Fregni F, Pagano RL (2011) Translational research in transcranial direct current stimulation (tDCS): a systematic review of studies in animals. Rev Neurosci 22:471-481. CrossRef Medline

Cancelli A, Cottone C, Di Giorgio M, Carducci F, Tecchio F (2015a) Personalizing the electrode to neuromodulate an extended cortical region. Brain Stimul 8:555-560. CrossRef Medline

Cancelli A, Cottone C, Zito G, Di Giorgio M, Pasqualetti P, Tecchio F (2015b) Cortical inhibition and excitation by bilateral transcranial alternating current stimulation. Restor Neurol Neurosci 33:105-114. CrossRef Medline

Cottone C, Porcaro C, Cancelli A, Olejarczyk E, Salustri C, Tecchio F (2017) Neuronal electrical ongoing activity as a signature of cortical areas. Brain Struct Funct 222:2115-2126. CrossRef Medline

Crochet S, Fuentealba P, Cissé Y, Timofeev I, Steriade M (2006) Synaptic plasticity in local cortical network in vivo and its modulation by the level of neuronal activity. Cereb Cortex 16:618-631. CrossRef Medline

de Pesters A, Coon WG, Brunner P, Gunduz A, Ritaccio AL, Brunet NM, de Weerd P, Roberts MJ, Oostenveld R, Fries P, Schalk G (2016) Alpha power indexes task-related networks on large and small scales: a multimodal ECoG study in humans and a non-human primate. Neuroimage 134:122-131. CrossRef Medline

Ecker AS, Berens P, Keliris GA, Bethge M, Logothetis NK, Tolias AS (2010) Decorrelated neuronal firing in cortical microcircuits. Science 327:584587. CrossRef Medline

Engel AK, Fries P, Singer W (2001) Dynamic predictions: oscillations and synchrony in top-down processing. Nat Rev Neurosci 2:704-716. CrossRef Medline

Fertonani A, Miniussi C (2016) Transcranial electrical stimulation: what we know and do not know about mechanisms. Neuroscientist. Advance online publication. Retrieved Feb. 12, 2016. CrossRef Medline

Fertonani A, Pirulli C, Miniussi C (2011) Random noise stimulation improves neuroplasticity in perceptual learning. J Neurosci 31:15416-15423. CrossRef Medline

Feurra M, Bianco G, Santarnecchi E, Del Testa M, Rossi A, Rossi S (2011) Frequency-dependent tuning of the human motor system induced by transcranial oscillatory potentials. J Neurosci 31:12165-12170. CrossRef Medline

Feurra M, Pasqualetti P, Bianco G, Santarnecchi E, Rossi A, Rossi S (2013) State-dependent effects of transcranial oscillatory currents on the motor system: what you think matters. J Neurosci 33:17483-17489. CrossRef Medline

Fröhlich F, McCormick DA (2010) Endogenous electric fields may guide neocortical network activity. Neuron 67:129-143. CrossRef Medline

Gal A, Marom S (2013) Entrainment of the intrinsic dynamics of single isolated neurons by natural-like input. J Neurosci 33:7912-7918. CrossRef Medline

Haegens S, Barczak A, Musacchia G, Lipton ML, Mehta AD, Lakatos P, Schroeder CE (2015) Laminar profile and physiology of the alpha rhythm in primary visual, auditory, and somatosensory regions of neocortex. J Neurosci 35:14341-14352. CrossRef Medline

Halliday DM, Rosenberg JR, Amjad AM, Breeze P, Conway BA, Farmer SF (1995) A framework for the analysis of mixed time series/point process data: theory and application to the study of physiological tremor, single motor unit discharges and electromyograms. Prog Biophys Mol Biol 64: 237-278. CrossRef Medline

Histed MH, Maunsell JH (2014) Cortical neural populations can guide behavior by integrating inputs linearly, independent of synchrony. Proc Natl Acad Sci U S A 111:E178-E187. CrossRef Medline

Jensen O, Gips B, Bergmann TO, Bonnefond M (2014) Temporal coding organized by coupled alpha and gamma oscillations prioritize visual processing. Trends Neurosci 37:357-369. CrossRef Medline

Kanai R, Chaieb L, Antal A, Walsh V, Paulus W (2008) Frequency-dependent electrical stimulation of the visual cortex. Curr Biol 18:1839-1843. CrossRef Medline

Kopell NJ, Gritton HJ, Whittington MA, Kramer MA (2014) Beyond the connectome: the dynome. Neuron 83:1319-1328. CrossRef Medline

Melgari JM, Zappasodi F, Porcaro C, Tomasevic L, Cassetta E, Rossini PM, Tecchio F (2013) Movement-induced uncoupling of primary sensory and motor areas in focal task-specific hand dystonia. Neuroscience 250: 434-445. CrossRef Medline

Moliadze V, Atalay D, Antal A, Paulus W (2012) Close to threshold transcranial electrical stimulation preferentially activates inhibitory networks before switching to excitation with higher intensities. Brain Stimul 5:505511. CrossRef Medline

Pascual-Marqui RD (2002) Standardized low-resolution brain electromagnetic tomography (sLORETA): Technical details, Methods Find. Exp. Clin. Pharmacol 24:5-12. Medline

Pittaccio S, Zappasodi F, Viscuso S, Mastrolilli F, Ercolani M, Passarelli F, Molteni F, Besseghini S, Rossini PM, Tecchio F (2011) Primary sensory and motor cortex activities during voluntary and passive ankle mobilization by the SHADE orthosis. Hum Brain Mapp 32:60-70. CrossRef Medline

Porcaro C, Tecchio F (2014) Semi-blind functional source separation algorithm from non-invasive electrophysiology to neuroimaging. In: Blind source separation: signals and communication technology (Wang GR, ed). Berlin, Germany: Springer.

Porcaro C, Barbati G, Zappasodi F, Rossini PM, Tecchio F (2008) Hand sensory-motor cortical network assessed by functional source separation. Hum Brain Mapp 29:70-81. CrossRef Medline

Renart A, de la Rocha J, Bartho P, Hollender L, Parga N, Reyes A, Harris KD (2010) The asynchronous state in cortical circuits. Science 327:587-590. CrossRef Medline

Tecchio F, Porcaro C, Barbati G, Zappasodi F (2007) Functional source separation and hand cortical representation for a brain-computer interface feature extraction. J Physiol 580:703-721. CrossRef Medline

Tecchio F, Zappasodi F, Porcaro C, Barbati G, Assenza G, Salustri C, Rossini PM (2008a) High-gamma band activity of primary hand cortical areas: a sensorimotor feedback efficiency index. Neuroimage 40:256-264. CrossRef Medline

Tecchio F, Zappasodi F, Pasqualetti P, De Gennaro L, Pellicciari MC, Ercolani M, Squitti R, Rossini PM (2008b) Age dependence of primary motor cortex plasticity induced by paired associative stimulation. Clin Neurophysiol 119:675-682. CrossRef Medline

Tecchio F, Cancelli A, Cottone C, Tomasevic L, Devigus B, Zito G, Ercolani M, Carducci F (2013) Regional personalized electrodes to select transcranial current stimulation target. Front Hum Neurosci 7:131. CrossRef Medline

Thut G (2014) Modulating brain oscillations to drive brain function. PLoS Biol 12:e1002032. CrossRef Medline

Thut G, Veniero D, Romei V, Miniussi C, Schyns P, Gross J (2011) Rhythmic TMS causes local entrainment of natural oscillatory signatures. Curr Biol 21:1176-1185. CrossRef Medline 